\title{
LA ACREDITACIÓN DE CENTROS DESTINADOS A PERSONAS MAYORES EN SITUACIÓN DE DEPENDENCIA: UN INSTRUMENTO AL SERVICIO DEL EMPLEO Y LA CALIDAD ASISTENCIAL
}

THE ACCREDITATION OF CENTRES FOR ELDERLY PEOPLE IN A SITUATION OF DEPENDENCY: A TOOL AT THE SERVICE OF EMPLOYMENT AND CARE QUALITY

FERNANDO JIMENO JIMÉNEZ

Funcionario público (IMSERSO).España 


\title{
RESUMEN
}

La presente investigación pretende analizar el desarrollo normativo autonómico sobre acreditación de centros residenciales y diurnos / nocturnos destinados a personas mayores en situación de dependencia, de manera concreta, en materia de recursos humanos. La acreditación se configura como instrumento de calidad asistencial y generación de empleo.

Se estudia el Acuerdo del Consejo Territorial del SAAD de 27/11/2008 (modificado parcialmente el 7/10/2015), donde se fijan criterios comunes sobre titulación y formación directiva, ratios mínimas de plantilla global y específica para Gerocultor/a o similar. Se configura la base normativa que cada Comunidad Autónoma deberá desarrollar o adaptar en el marco de su competencia.

Transcurrido el tiempo, se constata cómo el Acuerdo apenas ha sido desarrollado en cada ámbito territorial, se mantiene vigente en algunas autonomías la normativa preexistente y existe disparidad autonómica sobre requisitos de plantillas mínimas globales y específicas.

PALABRAS CLAVE: Acreditación, centros residenciales, centros diurnos / nocturnos, personas mayores, situación dependencia

\begin{abstract}
This research aims to analyse the regulatory development of the autonomous regions on the accreditation of residential centres and day/night centres for elderly people in a situation of dependency, specifically, in human resources. The accreditation is configured as an instrument of care quality and job creation.

It is studied the Agreement of the Territorial Council of the $S A A D$ of 27/11/2008 (partially modified on the 7/10/2015), where the common criteria on qualification and complementary training, minimum ratios of global and specific staff for geriatric caregivers or similar are established. The normative basis that each Autonomous Community will have to develop or adapt within the framework of their competence is configured.
\end{abstract}

As time has gone by, it is verified how the Agreement has barely been developed in each territorial area, the preexisting regulations are still in force in some of the autonomous regions and there is a disparity in the requirements of the minimum global and specific staff in the different autonomous regions.

KEYWORDS: Accreditation, residencial centres, day/night centres, elderly people, situation of dependency. 
I. EL SISTEMA DE DEPENDENCIA ESPAÑOL Y LA ACREDITACIÓN DE CENTROS

A. EL SISTEMA DE DEPENDENCIA ESPAÑOL Y LA PREFERENCIA POR LA ASIGNACIÓN DE SERVICIOS PROFESIONALES

B. DESARROLLO DEL ARTÍCULO 34.2 DE LA LAPAD: CRITERIOS COMUNES DEL CONSEJO TERRITORIAL EN MATERIA DE ACREDITACIÓN

C. CRITERIOS COMUNES DE ACREDITACIÓN EN MATERIA DE RECURSOS HUMANOS EN CENTROS PARA PERSONAS MAYORES

D. MISIÓN DEL ACUERDO DE CRITERIOS COMUNES DEL CONSEJO TERRITORIAL DE 27/11/2008

II. DESARROLLO NORMATIVO AUTONÓMICO DE LOS CRITERIOS COMUNES DE ACREDITACIÓN EN MATERIA DE RECURSOS HUMANOS EN CENTROS PARA PERSONAS MAYORES

III. CONCLUSIONES 


\section{EL SISTEMA DE DEPENDENCIA ESPAÑOL Y LA ACREDITACIÓN DE CENTROS}

\section{A. EL SISTEMA DE DEPENDENCIA ESPAÑOL Y LA PREFERENCIA POR LA ASIGNACIÓN DE SERVICIOS PROFESIONALES}

La aprobación de Ley 39/06, de promoción de la autonomía personal y atención a personas en situación de dependencia ${ }^{1}$ (LAPAD), supone un punto de inflexión en materia de cuidados de larga duración ${ }^{2}$, un intento de equiparación en materia de bienestar social con los países de nuestro entorno. El nuevo derecho subjetivo generado queda enmarcado en un modelo protector de naturaleza universal, con una tendencia nítida dirigida al fomento de la atención profesional y al respiro del cuidador tradicional. Implica un antes y un después, un cambio estructural superador de un sistema basado exclusivamente en actuaciones familiares.

Surgida la necesidad, el nuevo sistema de protección manifiesta una preferencia por la asignación de servicios como respuesta a la situación de dependencia. El artículo 14.4 de la LAPAD declara la excepcionalidad de la prestación económica para cuidados familiares frente a la actuación prioritaria de los servicios profesionales ${ }^{3}$.

¿Qué puede entenderse por servicio profesional? El potencial catálogo de servicios es definido en el artículo 15 de la Ley 39/06, incluyendo el tradicional acceso al (1) ingreso en centro residencial, el ampliamente demandando (2) servicio de ayuda a domicilio, (3) los centros diurnos / nocturnos, a mitad de camino entre los anteriores, (4) la teleasistencia domiciliaria, donde la tecnología facilita sus medios en apoyo del usuario y la detección de situaciones de urgencia y, finalmente (5) los programas de promoción y prevención de la autonomía, en fase de desarrollo. La (6) prestación económica vinculada al servicio y (7) la de asistente personal formarían parte del catálogo, todo vez que la primera se concede ante la ausencia de servicio público y demandar su prestación en el ámbito privado, y la segunda por tener como finalidad la promoción de la autonomía individual.

El nuevo modelo de protección pretende un avance en materia de calidad en la ejecución de las actuaciones y en el logro del objetivo de una gestión más eficaz (artículo 34.1 de la LAPAD), motivo por el que el legislador encomienda la elaboración de unos criterios homogéneos de acreditación al Consejo Territorial del Sistema para la Autonomía y Atención a la Dependencia (34.2), órgano de cooperación de composición paritaria Estado / Comunidades Autónomas (CC. AA.), todo ello, sin perjuicio de la competencia normativa propia de cada autonomía.

\footnotetext{
${ }^{1}$ Ley 39/06, de 14 de diciembre, publicada en el BOE de 15 de diciembre de 2006, núm. 299.

${ }^{2}$ La situación de dependencia queda definida por la valoración resultante de padecer limitaciones que impiden la realización de actos esenciales para la vida diaria y necesidad de tercera persona en la realización de cuidados.

${ }^{3}$ De acuerdo con la información suministrada por el Sistema de Dependencia del IMSERSO, en julio de 2017 dos de cada tres programas reconocidos son servicios $(66,45 \%)$, y el tercio restante $(33,55 \%)$ prestaciones económicas para cuidados familiares (consulta septiembre 2017). http://www.dependencia.imserso.es/InterPresent2/groups/imserso/documents/binario/estsisaad20170731. pdf.
}

e-Revista Internacional de la Protección Social, ISNN 2445-3269. 2017, Vol. II, No 2 
¿Qué es la acreditación de un centro o un servicio? La acreditación supone la comprobación por parte de la entidad gestora en materia de servicios sociales, las CC. AA. y el IMSERSO para Ceuta y Melilla, del cumplimiento de determinados requisitos y estándares de calidad (materiales y equipamiento / recursos humanos) y emisión, en su caso, de la posterior resolución de autorización administrativa.

\section{B. DESARrollo DEL ARTíCUlo 34.2 DE LA LAPAD: CRITERIOS COMUNES DEL CONSEJO TERRITORIAL EN MATERIA DE ACREDITACIÓN}

La habilitación del artículo 34.2 de la Ley 39/06 se ha plasmado en el Acuerdo del Consejo Territorial de 27/11/20084, donde se aprueban los criterios comunes de acreditación de centros y servicios. Se fija un doble ámbito de supervisión. Por una parte, los recursos humanos, fijando dotaciones mínimas en función de la tipología de centros y la intensidad de los cuidados, así como requisitos de titulación y la necesidad de programas de formación; por otra, los recursos materiales, equipamiento y documentación básica (reglamento régimen interior, plan de calidad, plan de emergencia, protocolos de actuación, condiciones de accesibilidad, aseguramiento de responsabilidad civil, carta de servicios, entre otros). No cabe duda que prima en la redacción una apuesta por calidad en el empleo y el fomento de la profesionalidad como instrumentos de mejora en la prestación de cuidados. Posteriormente el Acuerdo de $27 / 11 / 2008$ fue modificado por el $07 / 10 / 2015^{5}$ con la finalidad de adaptar los criterios aplicables al colectivo de Gerocultor/a y similares a la realidad laboral y educativa de esta importante categoría laboral.

La acreditación está dirigida a las entidades privadas. Tanto empresas como organizaciones sin ánimo de lucro ${ }^{6}$ son destinatarias últimas del proceso de verificación del cumplimiento de los estándares y requisitos de calidad. No es exigible la autorización administrativa en los centros públicos, por presuponer que superan los requisitos mínimos aunque, en el supuesto de falta de cumplimiento, quedan sometidos a la obligación de adaptarse.

¿Para qué sirve la acreditación? La obtención de la credencial por un centro o servicio no solo demuestra la certificación de un determinado nivel de calidad, sino que facilita la inclusión de la empresa en la red del sistema de dependencia. En este sentido, la acreditación es condición indispensable para actuar en dos ámbitos:

1- Por un lado, para que puedan las CC. AA. concertar plazas o servicios con entidades privadas (artículo 16.1 LAPAD)

2- Por otro, cuando el interesado pretenda acceder a la prestación económica vinculada al servicio ante la inexistencia de recurso público ${ }^{7}$.

\footnotetext{
${ }^{4}$ La Resolución de 02/12/2008 de la Secretaría de Estado de Política Social, Familias y Atención a la Dependencia y a la Discapacidad publica el Acuerdo del Consejo Territorial de 27/11/2008.

${ }^{5}$ La Resolución de 03/11/2015 de la Secretaría de Estado de Servicios Sociales e Igualdad publica el Acuerdo del Consejo Territorial de 07/10/2015.

${ }^{6}$ Tercer Sector en denominación del artículo 2.8 de la Ley 39/06.

${ }^{7} \mathrm{Si}$ las CC. AA. no disponen de plaza en centro o servicio propio o concertado, pueden reconocer la prestación económica vinculada al servicio. Su naturaleza finalista obliga al interesado a justificar con facturación el destino del importe concedido, pudiendo únicamente contratar la prestación de los servicios con entidad previamente acreditada.
}

e-Revista Internacional de la Protección Social, ISNN 2445-3269. 2017, Vol. II, No 2

http://dx.doi.org/10.12795/e-RIPS.2017.i02.09.

Página 118 
Por tanto, es la superación del filtro de la acreditación la que facilita al sector privado la inclusión en el marco de la LAPAD, debiendo para ello realizar un esfuerzo inversor en la mejora de infraestructuras ${ }^{8}$ y plantillas $\mathrm{Su}$ vigencia es temporal (4 años), con acotamiento territorial para cada autonomía.

Como muestra del ámbito potencial de actuación y de la trascendencia del proceso de acreditación, para el año 2014 son financiadas privadamente en España el 53\% de las plazas residenciales destinadas a personas mayores ${ }^{9}$, el $37 \%$ de las correspondientes a centros diurnos / nocturnos y la casi totalidad de la ayuda a domicilio, servicio sometido casi en exclusiva a externalización.

\section{CRITERIOS COMUNES DE ACREDITACIÓN EN MATERIA DE RECURSOS HUMANOS EN CENTROS PARA PERSONAS MAYORES}

En los párrafos anteriores se ha indicado la doble exigencia prevista en materia de acreditación, los requisitos materiales y los humanos. Las siguientes páginas se centrarán en las ratios mínimas en materia de personal que deben superarse para alcanzar la autorización administrativa, por referirse la presente exposición a la concreción autonómica en materia de empleo.

De igual forma, dada la amplitud que supondría, se pretende analizar el alcance que supone en el colectivo de personas mayores que reciben atención en centros asistenciales (residenciales y diurnos / nocturnos), justificando esta elección en el hecho de vincularse la situación de dependencia con el envejecimiento. Así, de conformidad con la información facilitada por el IMSERSO ${ }^{10}$ referida al mes de julio de 2017 , el $72,08 \%$ de las personas dependientes ha cumplido 65 o más años, donde el 54,43\% alcanza y supera la edad de 80 años.

En el desarrollo de este texto se analizará la incidencia de la acreditación en centros residenciales y diurnos / nocturnos. La residencia es alternativa a la permanencia en el domicilio familiar, facilita una atención integral del usuario durante toda la jornada, bajo un enfoque biopsicosocial, con servicios continuados de carácter personal y sanitario (artículo 25 LAPAD). El centro diurno / nocturno aplica el mismo diseño, aunque ceñido al marco temporal de la mañana (09.00 / 17:00, por lo general) o de la noche (a partir de las 21:00 / 22:00), con la finalidad de promover la autonomía y/o apoyar a los cuidadores familiares en tanto que programa de respiro (artículo 24 LAPAD.

\footnotetext{
8 Díaz Díaz, B. y García Ramos, R.; "La atención a la dependencia como motor de desarrollo económico", Documentación Social. 2015. n 177: 119-142.

${ }^{9}$ IMSERSO Observatorio de Personas Mayores (2015). "Las personas mayores en España. Informe 2014". Para el año 2014, 196.356 sobre un total de 381.480 plazas residenciales parapersonas mayores. En centros diurnos para mayores, 32.015 respecto de las 54.404 utilizadas. En el servicio de ayuda a domicilio, 340.960 usuarios con 65 y más años.

10 IMSERSO, Estadísticas del Sistema para la Autonomía y Atención a la Dependencia (consulta septiembre 2017).

http://www.dependencia.imserso.es/InterPresent2/groups/imserso/documents/binario/estsisaad20170731. pdf.
}

e-Revista Internacional de la Protección Social, ISNN 2445-3269. 2017, Vol. II, Nº 2 
El acuerdo del Consejo Territorial de 27/11/2008 incide en tres ámbitos en materia de empleo que deben cumplir los centros susceptibles de acreditación. Para ello fija unas condiciones mínimas circunscritas a:

1- La figura de la dirección del centro de mayores.

2- Una ratio global de plantilla.

3- Otra específica para cuidador/a, gerocultor/a o similar.

El incumpliendo de estos requisitos de plantilla mínima (dirección, global y específica de Gerocultor/a) implicaría no solo la denegación de la acreditación, sino la revocación de la misma una vez reconocida con motivo de algún seguimiento, lo que conllevaría la rescisión del concierto de plazas en su caso o la imposibilidad de justificar la prestación económica vinculada al servicio.

Es preciso tener en consideración, dado el volumen de empleo que generan los centros asistenciales, el contenido del artículo 38 de la Ley de Integración Social de los Minusválidos (LISMI), al establecer una reserva de obligado cumplimiento del $2 \%$ de trabajadores con discapacidad en aquellas empresas con al menos 50 empleados.

El Director/a del centro asistencial de mayores asume un papel preponderante tanto en la gestión interna, como la externa. Es responsable de su buen funcionamiento y organización, asumiendo la representación ante los servicios sociales públicos. Por este motivo se fija como criterio común la necesidad de estar en posesión de titulación universitaria (Licenciatura, Diplomatura o Grado), complementada con formación en materias relacionadas con la dependencia (discapacidad, geriatría, dirección de centros, gerontología, entre otras). Ante esta importante innovación, se habilita una excepción basada en la trayectoria profesional. Así, las personas que vengan ejerciendo las tareas de dirección durante un periodo mínimo de tres años de experiencia, a pesar de no poseer titulación universitaria, mantendrán su situación siempre que demuestren la formación complementaria.

Parecen razonables las exigencias formativas principal y complementaria en la dirección, especialmente ante la responsabilidad que implica su singular posición jurídica, como la derivada de la aplicación del artículo 757.3 de la Ley de Enjuiciamiento Civil (Ley 1/2000, de 7 de enero), donde se obliga a comunicar al Ministerio Fiscal situaciones de posible incapacitación, situación habitual en centros destinados a personas mayores dependientes. En el mismo sentido, la cautela prevista en el artículo 271.1 del Código Civil, al establecerse la necesidad de obtener autorización judicial previa al ingreso residencialcuandola persona está afectada por deterioro mental.

Cuestión menos pacífica es la referida a la exigencia de una ratio de plantilla general en residencias y centros diurnos / nocturnos, toda vez que al entrar en vigor la Ley 39/06 la media $^{11}$ existente era inferior a la prevista en el Acuerdo de 27/11/2008. La configuración global de un centro suele estar determinada por la dirección, administración, puestos de atención directa al usuario (sociosanitarios), hostelería,

\footnotetext{
${ }^{11}$ De conformidad con el Acuerdo de 27/11/2008, la plantilla media en centros residenciales de personas mayores se situaba entre 4 y 6 puntos porcentuales inferior a la ratio a implantar, motivo por el que se facilita un periodo transitorio hasta 2011. La prevista para centros diurnos/nocturnos estaba más ajustada.
}

e-Revista Internacional de la Protección Social, ISNN 2445-3269. 2017, Vol. II, Nº 2 
limpieza y mantenimiento de instalaciones. Aunque no esté incluida en la ratio mínima de plantilla, no debe descartarse la importancia que adquieren las empresas suministradoras de alimentos y otros bienes ${ }^{12}$.

Tabla 1: Ratios de plantilla global mínimas y simulación(elaboración propia)

\begin{tabular}{|c|c|c|c|}
\hline & $\begin{array}{c}\text { RATIO } \\
\text { GRADO II }\end{array}$ & $\begin{array}{c}\text { RATIO } \\
\text { GRADO III }\end{array}$ & $\begin{array}{c}\text { SIMULACIÓN } \\
\text { PLANTILLA MÍNIMA }\end{array}$ \\
\hline $\begin{array}{c}\text { RESIDENCIA MAYORES } \\
(100 \text { personas GRADO III y } 40 \\
\text { GRADO II) }\end{array}$ & 0,45 & 0,47 & $\begin{array}{c}65 \text { empleados } \\
\text { (65 jornadas completas) }\end{array}$ \\
\hline $\begin{array}{c}\text { CENTRO DÍA MAYORES } \\
\text { (20 personas GRADO II) }\end{array}$ & 0,23 & 0,24 & $\begin{array}{c}4,6 \\
(4 \text { jornadas completas y } \\
24 \text { horas semanales })\end{array}$ \\
\hline
\end{tabular}

De conformidad con lo indicado en la tabla 1 se fijan dos ratios para cada centro en función de la situación de dependencia que tenga reconocida cada beneficiario. Para el grado II (dependencia severa) se establece una plantilla mínima en centros residenciales del $45 \%$ sobre el número de usuarios y del $47 \%$ para grado III (gran dependencia). En relación con los centros diurnos / nocturnos, $23 \%$ y $24 \%$ respectivamente.

Deben efectuarse dos matizaciones. Por una parte, el sistema de dependencia español solo permite el ingreso residencial para grados II y III, añadiendo el grado I (dependencia moderada) para los centros diurnos / nocturnos. Por tanto, se detecta una laguna en la fijación de la dotación de personal en los centros de día / noche cuando los usuarios tienen reconocido grado I, supuesto muy habitual en esta tipología de centros. Por otra, a los efectos del cómputo, se incluye a la totalidad de personas que prestan servicios en los centros, con independencia de la modalidad contractual, laboral o mercantil, tomando como referencia la jornada prevista en el convenio colectivo (1792 horas anuales para centros asistenciales ${ }^{13}$ ).

Con los parámetros reflejados en la tabla 1, un centro residencial con 100 beneficiarios en situación de gran dependencia y 40 con severa necesitaría al menos una plantilla de 65 personas a jornada completa, incluyendo la figura del Director/a en la misma. El centro diurno con 20 usuarios grado II, un mínimo de 4 trabajadores a tiempo completo y 24 horas semanales. En el supuesto, muy habitual, del centro residencial anterior con unidad de estancias diurnas, se realizaría sumatorio (69 trabajadores y 24 horas semanales), siendo posible de conformidad con la normativa autonómica que la dirección se común.

Dentro de esa plantilla global estaría incluido el colectivo de Gerocultores/as, el grupo más numeroso que presta servicios en el centro, sobre el que se establece un porcentaje específico. Para realizar sus tareas deberán estar en posesión de la cualificación en

\footnotetext{
${ }^{12}$ Rodríguez Castedo, Á. y Jiménez Lara, A.; "La atención a la dependencia y el empleo". Laboratorios de Alternativas, Documentos de trabajo 159/2010.

13 Artículo 37 del convenio colectivo marco estatal de servicios de atención a personas dependientes y desarrollo de la promoción de la autonomía personal, publicado en el BOE de 18/05/2012, núm.119, posteriormente con modificación en las tablas salariales.
} 
Atención Sociosanitaria a Personas Dependientes en Instituciones Sociales (Real Decreto 1368/2007), aunque se establecen asimilaciones y equivalencias ${ }^{14}$. Como quiera que existe un importante núcleo de personas sin titulación, se ha previsto el denominado proceso de habilitación por parte de los Servicios Sociales autonómicos entre los Gerocultores/as con edad de 55 años o más (a 31/12/2015) y tres años de experiencia en la categoría profesional, necesitando acreditar la realización de las funciones durante 2.000 horas en los últimos diez años. En todo caso, se ha fijado el 31/12/2017 como fecha de cierre para obtener la cualificación por alguna de estas modalidades.

En la tabla 2 se reflejan las ratios mínimas previstas para Gerocultores/as. De manera detallada, en los centros residenciales el $28 \%$ y $27 \%$ respectivamente para grados III y II. En centros diurnos / nocturnos, $15 \%$ y $14 \%$. Aplicando la misma ocupación de plazas que en el ejemplo de la tabla 1, sería preciso el empleo de 38 Gerocultores/as a jornada completa y 32 horas semanales adicionales para un centro residencial con 100 beneficiarios grado III y 40 con dependencia severa. En el centro diurno, 2 profesionales y 32 horas semanales para atender a 20 usuarios grado II. Como se ha comentado con anterioridad, la categoría profesional de Gerocultores/as presenta tratamiento específico, pero a la vez forma parte de la plantilla global del centro.

Tabla 2: Ratios específicas de plantilla mínima para Gerocultores/as y simulación (elaboración propia)

\begin{tabular}{|c|c|c|c|}
\hline & $\begin{array}{c}\text { RATIO } \\
\text { GRADO II }\end{array}$ & $\begin{array}{c}\text { RATIO } \\
\text { GRADO III }\end{array}$ & $\begin{array}{c}\text { SIMULACIÓN } \\
\text { PLANTILLA MÍNIMA }\end{array}$ \\
\hline $\begin{array}{c}\text { RESIDENCIA MAYORES } \\
(100 \text { personas GRADO III y 40 } \\
\text { GRADO II) }\end{array}$ & 0,27 & 0,28 & $\begin{array}{c}38,8 \text { empleados } \\
\text { (38 jornadas completas y } \\
32 \text { horas semanales })\end{array}$ \\
\hline $\begin{array}{c}\text { CENTRO DÍA MAYORES } \\
\text { (20 personas GRADO II) }\end{array}$ & 0,14 & 0,15 & $\begin{array}{c}2,8 \text { empleados } \\
(2 \text { jornadas completas y } \\
32 \text { horas semanales })\end{array}$ \\
\hline
\end{tabular}

Con la finalidad de valorar la importancia del empleo residencial en España y resaltar la importancia que adquieren los criterios anteriores de contratación laboral, en el gráfico 1 se representa la evolución de población activa en este sector en el periodo 2008/2017. En el segundo trimestre del año 2017 unas 300.000 personas se encuentran trabajando en las residencias españolas, con un predominio femenino cercano al $87 \%$. De igual forma, se detecta cómo ha crecido en un 50\% la plantilla en un periodo de nueve años, circunstancia imputable al desarrollo del sistema de dependencia. Otro aspecto destacable es el referido a la menor incidencia en este sector de la crisis económica iniciada en el 2007, toda vez que solamente se atisba disminución en los años 2012 y 2013. Todo ello viene a demostrar la importancia que adquiere un servicio necesario en la población española como es el residencial, con una demanda de ocupación elevada con respecto a nuestro entorno europeo.

\footnotetext{
${ }^{14}$ Se asimilan los títulos y certificados: (1)Técnico en Cuidados Auxiliares de Enfermería (Real Decreto 546/1995), (2)Técnico de Atención a Personas en Situación de Dependencia (Real Decreto 1593/2011), (3) Técnico de Atención Sociosanitaria (Real Decreto496/2003) o las equivalencias fijadas en el Real Decreto 777/1998. De igual modo, el Certificado de Profesionalidad.
}

e-Revista Internacional de la Protección Social, ISNN 2445-3269. 2017, Vol. II, N 2 
Para finalizar este apartado, debe efectuarse alguna reflexión final. Es cierto que la elaboración de estos criterios supone una avance sustancial homogeneizador en un área de especial sensibilidad social. La regulación de determinadas titulaciones profesionales y el cumplimiento de porcentajes de empleo en función del número de usuarios presentan un itinerario en dirección hacia la calidad en la ejecución de las tareas.

Gráfico 1: Ocupación laboral en centros residenciales ${ }^{\mathbf{1 5}}$

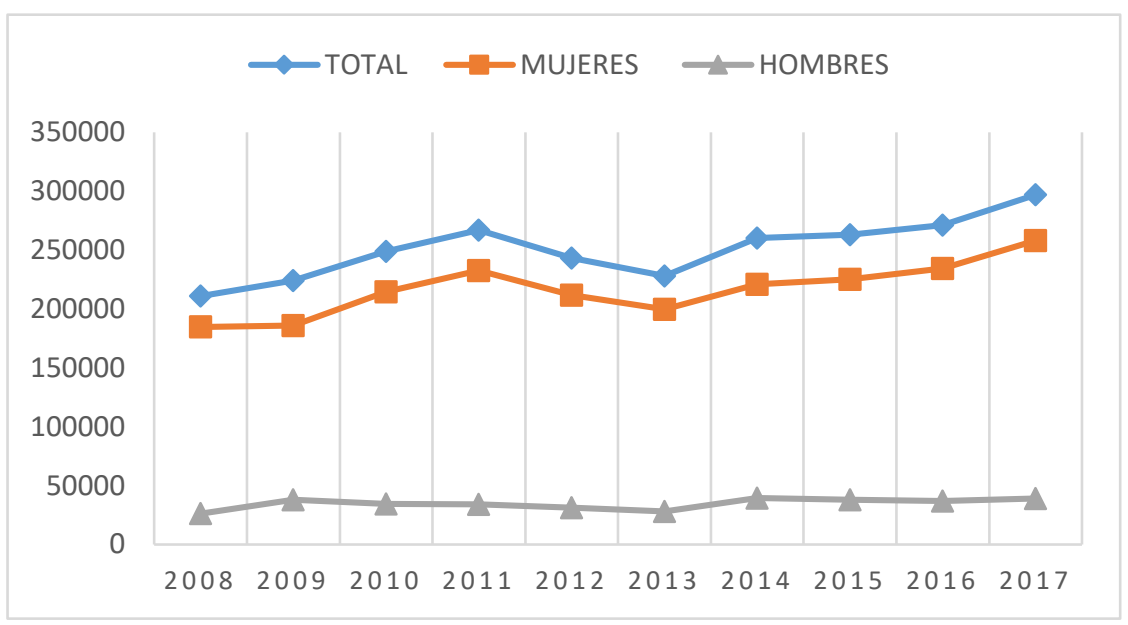

No obstante, hubiera sido el momento no sólo de regular la plantilla global, la particular de cuidadores y los requisitos de la dirección del centro, sino de afrontar la configuración de los restantes puestos de atención directa (Medicina, Psicología, Enfermería, Fisioterapia, Terapia Ocupacional, Trabajo Social, Educación Social, Logopedia). Como muestra, siguiendo el ejemplo simulador del centro residencial anterior, desde la perspectiva de recursos humanos puede acreditarse el centro si la plantilla alcanza 65 empleados, entre los que se encuentren la figura del Director/a y 38 Gerocultores/as con 32 horas semanales adicionales, pudiendo completarse el resto con trabajadores de servicios generales (hostelería, limpieza o mantenimiento). No se obliga a la cobertura de puestos de atención directa esenciales en el devenir de unas instalaciones de atención integral socio sanitaria (titulados/as universitarios).

\section{MISIÓN DEL ACUERDO DE CRITERIOS COMUNES DEL CONSEJO TERRITORIAL DE 27/11/2008}

De conformidad con lo previsto en los artículos 16 y 34.2 de la Ley 39/06, las CC. AA. y el IMSERSO para Ceuta y Melilla son competentes en materia de acreditación de centros y servicios, correspondiendo a cada una el desarrollo normativo, reglamentación y ejecución en el marco de los criterios comunes del Acuerdo del Consejo Territorial de 27/11/2008 y su modificación parcial de 7/10/2015.

El Acuerdo sobre criterios comunes cumple una función de homogenización y fijación de mínimos a desarrollar en cada ámbito territorial por las CC. AA. e IMSERSO. No es directamente aplicable, ni exigible jurídicamente. Su cometido pretende facilitar bases

${ }^{15}$ Elaborado con información obtenida del INE (Instituto Nacional de Estadística), Encuesta de Población Activa. Datos referidos al segundo trimestre de cada anualidad (consulta septiembre 2017). http://www.ine.es/jaxiT3/Tabla.htm?t=4200\&L=0

e-Revista Internacional de la Protección Social, ISNN 2445-3269. 2017, Vol. II, N 2 
armonizadas de elaboración normativa, competencia propia autonómica. La LAPAD facilita un proceso descentralizador en materia de servicios sociales, donde las CC. AA. tienen amplia capacidad de desarrollo normativo ${ }^{16}$ en el marco de la propia Ley 39/06 y los criterios aprobados.

En este sentido se manifiesta la Sentencia de la Audiencia Nacional (Sala de lo Contencioso Administrativo) de 25/02/2011, tras recurso (90/2009) promovido por el Comité Español de Representantes de Personas con Discapacidad (CERMI) contra Acuerdo del Consejo Territorial en materia de determinación de la capacidad económica del beneficiario y criterios de participación en las prestaciones del sistema de dependencia, al indicar:

1- El Consejo Territorial tiene una capacidad de propuesta dirigida a los órganos competentes.

2- Elabora criterios para que sean trasladados a forma reglamentaria.

Expuesto lo anterior, es el momento de efectuar análisis de cómo las CC. AA. y el IMSERSO (para las Ciudades Autónomas) han desarrollado los criterios comunes en su ámbito territorial, con examen de las principales particularidades.

\section{DESARROLLO NORMATIVO AUTONÓMICO DE LOS CRITERIOS COMUNES DE ACREDITACIÓN EN MATERIA DE RECURSOS HUMANOS EN CENTROS PARA PERSONAS MAYORES}

En la tabla 3 se exponen los principales textos normativos elaborados por las CC. AA. e IMSERSO en el desarrollo de su competencia sobre acreditación de centros destinados a personas mayores (residenciales y diurnos / nocturnos).

Del análisis normativo se aprecian actuaciones en una doble dimensión:

1- Se constata como todas las autonomías tienen aprobados procedimientos reglamentarios (decretos, órdenes o resoluciones) sobre autorización o acreditación de centros asistenciales, en un importante número de fecha anterior al Acuerdo del Consejo Territorial de 27/11/2008, lo que motiva la ausencia de algunos de sus compromisos (carta de servicios o referencias a programas de calidad).

2- Sin embargo, sólo tres CC. AA. han aprobado normas donde asumen los criterios comunes en materia de recursos humanos y sus porcentajes de plantilla mínima (Asturias, Baleares e IMSERSO). Extremadura asume los parámetros condicionando su aplicación desarrollo normativo.

Es habitual el recurso a conceptos indeterminados en la configuración de la plantilla, utilizando expresiones como personal suficiente (Cantabria o Cataluña) para la correcta atención (Valencia) y con titulación adecuada (Andalucía), con remisión a la tipología del centro y la intensidad de los servicios a prestar (Baleares) o plantilla precisa para garantizar atención y calidad (Murcia). De igual forma, se produce en determinados

\footnotetext{
${ }^{16}$ López Casasnovas, G. y Del Pozo Rubio, R.; "La protección social de los problemas de dependencia en España”. Documentos de Trabajo CRES - Fundación Caser. 2010. (1).
}

e-Revista Internacional de la Protección Social, ISNN 2445-3269. 2017, Vol. II, No 2 
supuestos reenvío específico al contenido de los pliegos de prescripciones técnicas (Aragón o Cataluña), donde se concretan en detalle las ratios exigibles.

Tabla 3: Referencia a principal normativa autonómica en materia de acreditación de centros residenciales y diurnos/ nocturnos para personas mayores ${ }^{17}$ (elaboración propia)

\begin{tabular}{|c|c|}
\hline ANDALUCÍA & $\begin{array}{l}\text { Orden de } 5 / 11 / 2007(14 / 11 / 2007) \\
\text { Orden } 22 / 09 / 2008(07 / 10 / 2008) \\
\text { Orden } 1 / 7 / 1997(15 / 07 / 1997)\end{array}$ \\
\hline ARAGÓN & $\begin{array}{c}\text { Decreto } 111 / 1992,26 \text { de mayo }(10 / 06 / 1992) \\
\text { Orden } 19 / 03 / 1998(08 / 04 / 1998)\end{array}$ \\
\hline $\begin{array}{l}\text { PRINCIPADO DE } \\
\text { ASTURIAS }\end{array}$ & $\begin{array}{c}\text { Decreto 43/2011, de } 17 \text { de mayo (04/06/2011) } \\
\text { Res. 25/11/2011 (28/11/2011) }\end{array}$ \\
\hline ISLAS BALEARES & $\begin{array}{c}\text { Decreto 86/2010, de } 25 \text { de junio }(03 / 07 / 2010) \\
\text { Decreto } 54 / 2013 \text {, de } 5 \text { de diciembre }(07 / 12 / 2013)\end{array}$ \\
\hline CANARIAS & $\begin{array}{c}\text { Decreto 67/2012, de } 20 \text { de junio (13/08/2012) } \\
\text { Resolución 28/05/2013 }\end{array}$ \\
\hline CANTABRIA & $\begin{array}{c}\text { Orden EMP } 37 / 2010,18 \text { de marzo (06/04/2010), posteriormente } \\
\text { modificada por Orden EMP 21/2011, Orden SAN 39/2012 y Orden } \\
\text { MED 13/2015 }\end{array}$ \\
\hline CATALUÑA & $\begin{array}{l}\text { Ordre } 35 / 2014, \text { de } 20 \text { de febrero }(26 / 02 / 2014) \\
\text { Decreto } 176 / 2000, \text { de } 15 \text { de mayo }(26 / 05 / 2000)\end{array}$ \\
\hline CASTILLA LA MANCHA & $\begin{array}{c}\text { Orden 04/06/2013, (12/06/2013) } \\
\text { Orden de 21/05/2001 (29/06/2001) } \\
\end{array}$ \\
\hline CASTILLA y LEÓN & Decreto $2 / 2016,4$ de febrero $(05 / 02 / 2016)$ \\
\hline EXTREMADURA & Decreto $298 / 2015$, de 20 de noviembre $(26 / 11 / 2015)$ \\
\hline GALICIA & $\begin{array}{l}\text { Decreto } 149 / 2013 \text {, de } 5 \text { de septiembre (24/09) que remite a la Orden de } \\
\qquad 18 / 04 / 1996(06 / 05 / 1996)\end{array}$ \\
\hline LA RIOJA & $\begin{array}{l}\text { Decreto } 27 / 1998, \text { de } 6 \text { de marzo }(07 / 03 / 1998) \\
\text { Decreto } 12 / 1993 \text {, de } 18 \text { de febrero }(25 / 02 / 1993)\end{array}$ \\
\hline $\begin{array}{l}\text { COMUNIDAD DE } \\
\text { MADRID }\end{array}$ & Decreto $21 / 2015$, de 16 de abril $(17 / 04 / 2015)$ \\
\hline REGIÓN DE MURCIA & $\begin{array}{l}\text { Decreto } 3 / 2015 \text {, de } 23 \text { de enero }(27 / 01 / 2015) \\
\text { Decreto } 69 / 2005 \text {, de } 3 \text { de junio }(13 / 06 / 2005)\end{array}$ \\
\hline $\begin{array}{l}\text { COMUNIDAD FORAL DE } \\
\text { NAVARRA }\end{array}$ & Decreto Foral 209/1991, de 23 de mayo (05/07/1991) \\
\hline PAÍS VASCO & $\begin{array}{c}\text { Decreto 40/1998, de } 10 \text { de marzo }(02 / 04 / 1998) \\
\text { Decreto 41/1998, de } 10 \text { de marzo }(07 / 04 / 1998) \\
\text { Decreto } 202 / 2000 \text {, de } 17 \text { de octubre }(08 / 11 / 2000)\end{array}$ \\
\hline $\begin{array}{l}\text { COMUNIIDAD } \\
\text { VALENCIANA }\end{array}$ & $\begin{array}{l}\text { Orden } 04 / 02 / 2005(14 / 02 / 2005) \\
\text { Sobre autorización y funcionamiento de centro para mayores }\end{array}$ \\
\hline IMSERSO (Ceuta & Orden SAS 2287/2010, 19 agosto (30/08/2010) \\
\hline
\end{tabular}

La figura del Director/a del centro de mayores no se regula en todas las CC. AA., detectando cómo el desarrollo normativo ha planteado una variedad de regulaciones:

1- En algunas autonomías la jornada se fija en función del número de beneficiarios. Castilla y León obliga a jornada completa cuando se superan los 60 usuarios y Andalucía admite la posibilidad de compartir funciones directivas en centros residenciales con diurnos en el mismo edificio, exigiendo a cambio dedicación total.

\footnotetext{
${ }^{17}$ Se ha comprobado la información en junio de 2017. Entre paréntesis se indica la fecha de publicación en el diario oficial.
}

e-Revista Internacional de la Protección Social, ISNN 2445-3269. 2017, Vol. II, Nº 2 
2- La formación complementaria no se tiene en cuenta cuando la normativa aplicable preexiste al Acuerdo del Consejo Territorial de 27/11/2008.

3- En Cantabria se establece el lugar de impartición de los cursos complementarios y en Castilla la Mancha se pueden suplir por un periodo de un año de antigüedad.

4- Cataluña fija como preferentes las titulaciones en Ciencias de la Salud o Sociales para la dirección en centros diurnos.

5- Madrid ha creado un registro de directores/as de centros de servicios sociales, al igual que Castilla y León.

6- Andalucía concreta la intensidad de la formación complementaria en 300 horas.

No todas las CC. AA. fijan las ratios mínimas global de plantilla residencial y diurna / nocturna. Entre las que sí han regulado, unas reproducen los parámetros del Acuerdo de 27/11/2008 (Asturias y e IMSERSO para Ceuta y Melilla), llegando otras incluso a superar los porcentajes (Baleares y Andalucía).

Los mínimos de plantilla en el colectivo de gerocultores/as o similares son de difícil comprobación, toda vez que algunas CC. AA. han preferido fijar proporciones para el personal de atención directa, donde no sólo se englobaría a los Gerocultores/as, sino también a categorías sociales y sanitarias (Medicina, Psicología, Enfermería, Fisioterapia, Terapia Ocupacional, Trabajo Social o Educación Social). A continuación se destacan algunos aspectos por CC. AA.:

1- Asturias e IMSERSO reproducen las ratios mínimas exigibles para Gerocultores/as y similares.

2- Baleares obliga a porcentajes superiores $(0,28 \%$ y $0,15 \%$ para residencias y centros diurnos / nocturnos respectivamente).

3- En algún supuesto se regula en función de la categoría del centro (La Rioja) o de horas por día / noche o semana (Cantabria).

4- Aunque es un aspecto no recogido en el Acuerdo del Consejo Territorial de 27/11/2008, es frecuente que las CC. AA. establezcan ratios específicas para las categorías con titulación universitaria (Andalucía, Aragón, Cataluña, Castilla la Mancha, Galicia, La Rioja, Murcia o Navarra).

5- Navarra reconoce la figura del profesional de referencia, cada vez más demandado en estudios de investigación ${ }^{18}$, al igual que en Castilla y León.

6- Con la finalidad de mejorar actividades de participación y dinamización, emergen categorías como la de Monitor y Animador Socio Cultural (Asturias).

Para finalizar este apartado, es preciso reflexionar en torno a la necesidad de efectuar el control de plantilla de un centro susceptible de acreditar, no sólo con motivo de la presentación de solicitud, sino de manera periódica una vez operativa. No cabe duda que el número de beneficiarios puede variar y con las oscilaciones se modifica el resultado de la proporción. Los planes de inspección se estiman imprescindibles en la comprobación de la calidad asistencial ${ }^{19}$.

\footnotetext{
${ }^{18}$ Rodríguez Rodríguez, P.; "El sistema de servicios sociales español y las necesidades derivadas de la atención a la dependencia". Laboratorio de Alternativas (Fundación Alternativas), Documento de trabajo $87 / 2006$

${ }^{19}$ A modo de ejemplo, la Comunidad Autónoma de Navarra tiene regulado por Orden de la Consejería de Políticas Sociales 260/2015, de 27 de abril, BO de Navarra del 29 de mayo, núm. 103, su Plan de Inspecciones.
}

e-Revista Internacional de la Protección Social, ISNN 2445-3269. 2017, Vol. II, Nº 2 


\section{CONCLUSIONES}

El artículo 34.2 de la Ley 39/06, reguladora del sistema de dependencia español, configura la acreditación en tanto que instrumento destinado a fomentar el empleo y la calidad asistencial de centros y servicios. El legislador emite un mandato al Consejo Territorial con la finalidad de elaborar criterios comunes materiales y de recursos humanos que sirvan de base al posterior desarrollo normativo por las CC. AA.

El papel que deben asumir las CC. AA. en materia de acreditación es relevante por afectar a un importante yacimiento de empleo, la prestación de cuidados de larga duración. Muestra de ello, para el supuesto residencial de personas mayores, en el periodo 2008 /2017 la ocupación laboral se ha incrementado en torno al 50\%, con una valoración especial por coincidir con ciclo de crisis económica.

El acuerdo del Consejo Territorial de 27/11/2008 (posteriormente modificado el 07/10/2015), en matera de empleo, se centra en fijar requisitos de titulación académica y formación complementaria en la dirección, en ratios de plantilla global mínima y para la categoría de gerocultor/a o similar, la más numerosa en un centro para personas mayores dependientes. Se ha perdido la oportunidad de establecer criterios mínimos en los restantes puestos de atención directa, los que requieren titulación universitaria de naturaleza social o sanitaria, indispensables en un marco de calidad asistencial.

Como quiera que los criterios comunes fijados por el Consejo Territorial constituyen una propuesta homogeneizadora, mínimos a desarrollar normativamente por las CC. AA., titulares de la competencia, se examina la elaboración reglamentaria de cada una con referencia a centros residenciales y diurnos / nocturnos para personas mayores en situación de dependencia.

Aunque las CC. AA. tienen elaborados procedimientos de autorización / acreditación de centros para mayores, los cimientos creados por el Consejo Territorial en materia de empleo apenas han sido desarrollados. Solamente cuatro autonomías reproducen en su ámbito competencial los criterios mínimos de plantilla, manteniendo algunas su normativa preexistente. Se aprecia una regulación heterogénea, alejada del pretendido ideal de homogeneidad, con disparidad en la concreción de ratios mínimas, incluso con lagunas en la configuración de requisitos.

Se estima positivo que las CC. AA. hayan regulado porcentajes mínimos de plantilla con titulación universitaria, puestos de trabajo denominados de atención directa, toda vez que supone un avance en dirección a la mejora de la calidad del servicio a prestar. 